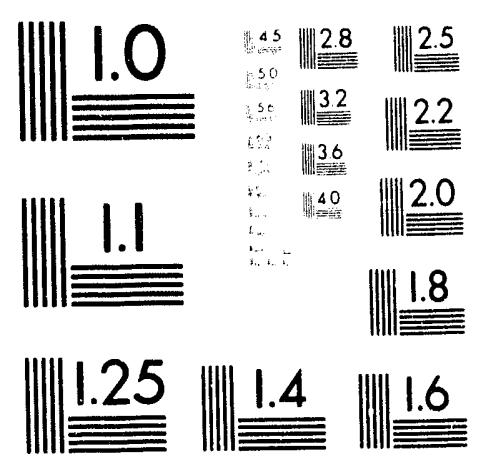



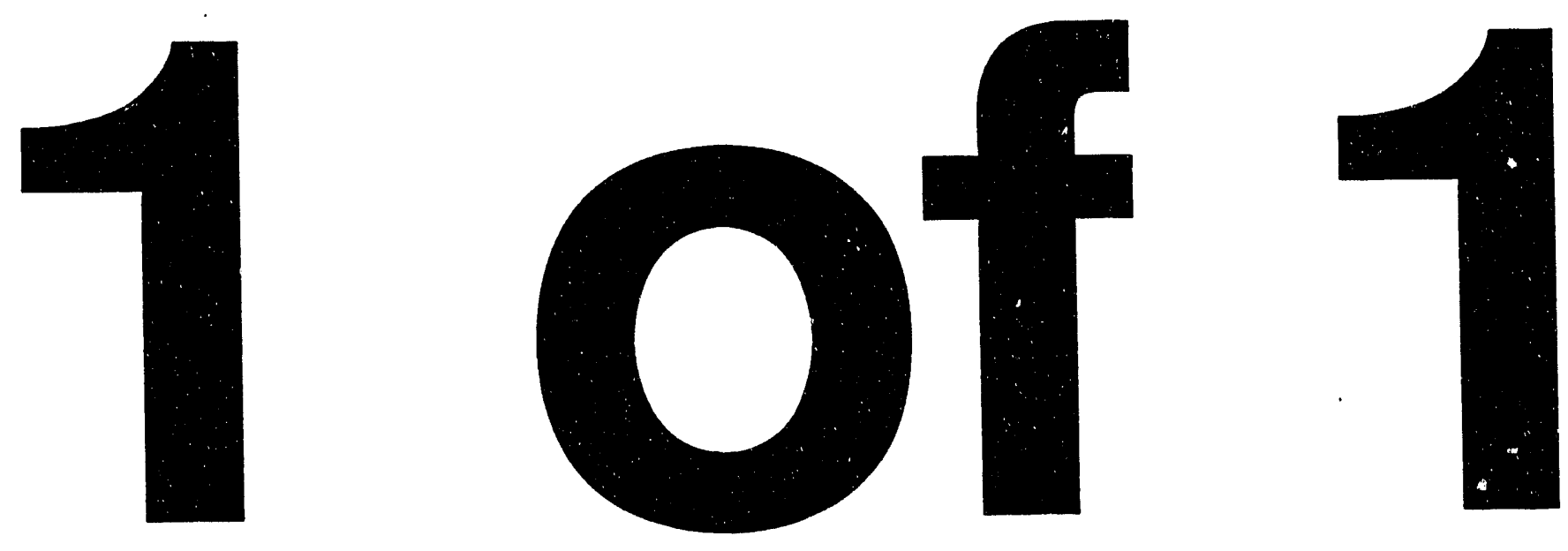
UCRL-CR-114650

B160610

\title{
Program PSNN
}

\author{
W.L. Morgan \\ Kinema Research \\ and \\ J.T. Larsen
}

Cascade Applied Sciences, Inc.

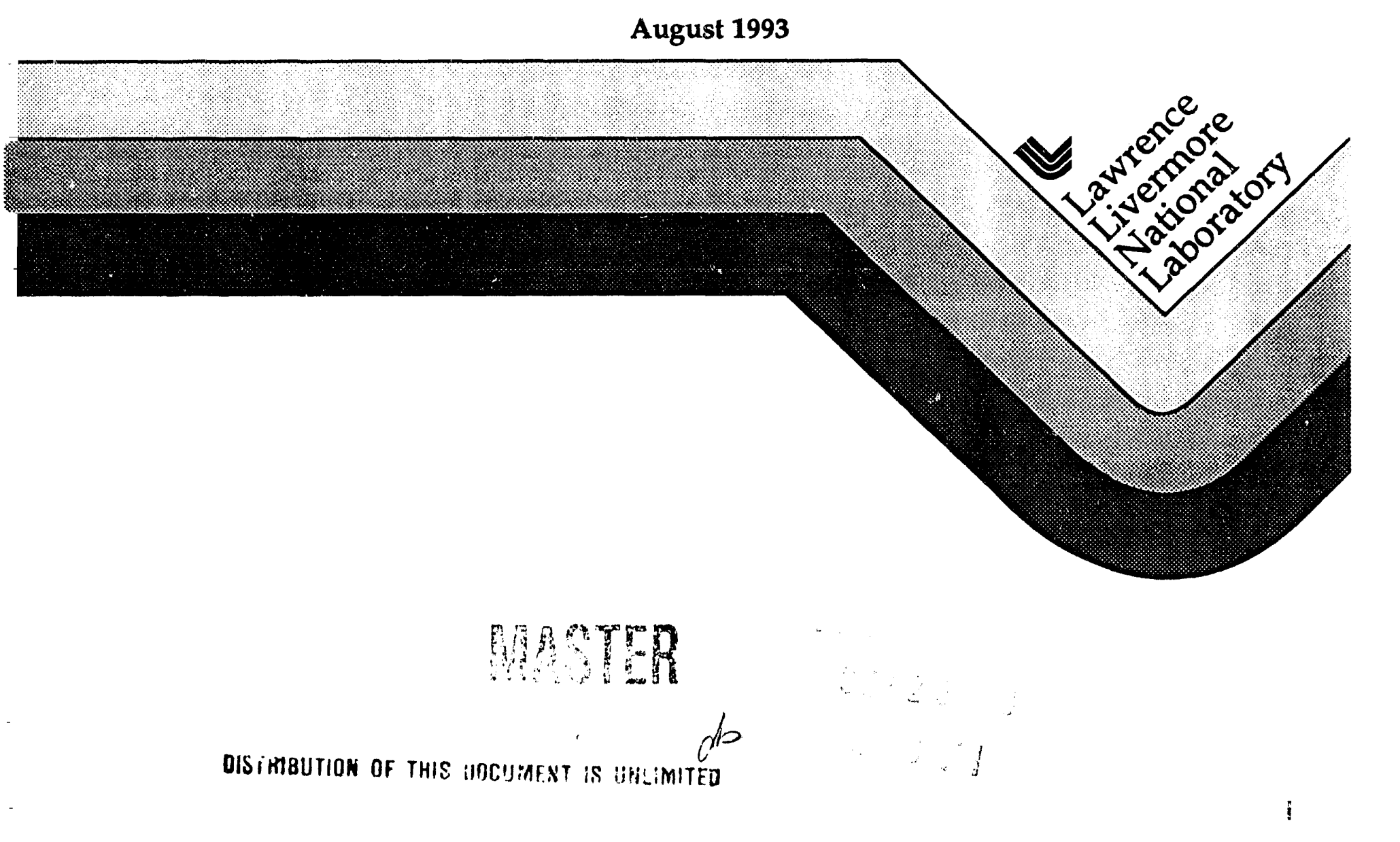




\section{DISCI.AIMER}

Work performed under the auspices of the U.S. Department of Energy by Lawrence Livermore National Lahoratory under contract number W-7405-ENG-48.

This document was prepared as an account of work sponsored by an agency of the United States Government. Neither the United States Government nor the University of California nor any of their employees, makes any warranty, express or implied, or assumes any legal liability or responsibility for the accuracy, completeness, or usefulness of any information. apparatus, product, or process disclosed. or represents that its use would not infringe privately owned rights. Reference herein to any specific commercial products, process, or service by trade name, tradernark, manufacturer, or otherwise, does not necessarily constitute or imply its endorsement, recommendation, or favoring by the United States Government or the University of California. The views and opinions of authors expressed herein do not necessarily state or reflect those of the United States Government or the University of California, and shall not be used for advertising or product endorsement purposes. 


\title{
PROGRAM PSNN
}

\section{Plasma Spectroscopy Neural Network}

\author{
W.L. Morgan \\ Kinema Research \\ 18720 Autumn Way, Monument, CO 80132 \\ Tel: (719) 488-3187; Fax: (719) 481-9140 \\ E-Mail: morgan@jiladc.colorado.edu
}

\section{J.T. Larsen}

Cascade Applied Sciences, Inc

P.O. Box 4477

Boulder, CO 80306

Tel, Fax: (303) 786-8990

E-Mail: larsen@ocfmail.ocf.llnl.gov

\section{Summary}

This program uses the standard "delta rule" back-propagation supervised training algorithm for multi-layer neural networks. The inputs are line intensities in arbitrary units, which are then normalized within the program. The outputs are $\mathrm{T}_{\mathrm{e}}(\mathrm{eV}), \mathrm{N}_{\mathrm{e}}$ $\left(\mathrm{cm}^{-3}\right)$, and a fractional ionization, which in our testing using $\mathrm{H}$ - and $\mathrm{He}-$ like spectra, was $\mathrm{N}(\mathrm{He}) /[\mathrm{N}(\mathrm{H})+\mathrm{N}(\mathrm{He})]$. 


\section{NEURAL NETWORKS}

\subsection{Neural Network Architecture}

Based on notions of how human brains are structured artificial neural networks (ANN) consist of layers of simulated "neurons" with associated activation functions, transfer functions, and weighting functions for the "synapse" connections to other neurons. A typical configuration for an ANN is shown in Fig. 1. The key elements are an input layer, one or more "hidden" layers, and an output layer. Each neuron has a transfer function associated with it, typically the sigmoid function $T(x)=1 /\left(1+e^{-x}\right)$, that gives an output value that is a non-linear function of the sum of the input values. The input values are the weighted outputs of each neuron in the previous layer. That is, if the output of neuron $j$ is $o_{j}$ and $w_{i j}$ is the weight connecting neurons $i$ and $j$, then the output of neuron $i$ is:

$$
o_{i}=T\left(\Sigma_{j} w_{i j} o_{j}\right)=1 /\left(1+e^{-\Sigma w_{i j} o_{j}}\right)
$$

where the sum is over all neurons $\mathrm{j}$ having outputs that feed into neuron $\mathrm{i}$. The hidden layers give the network a high degree of non-linearity and, from the point of view of the network as a pattern matcher, provide an internal representation of the correlation between the input and the output patterns. The concept behind this kind of network (known as a feed-forward, back-propagation network) is that it can "learn" to associate a set of output patterns with a set of input patterns by adjusting the weights that connect together the network of non-linear devices. One might also think of a neural network as a highly flexible many parameter interpolation and, hence, extrapolation algorithm. 


\subsection{Training Neural Networks}

Neural networks have a "learning" capability in that the weights associated with connections between pairs of neurons can be modified (strengthened or weakened) in response to the network's successes and failures so as to optimize in favor of the network's successful strategies. The network is trained by running a number of cases of known \{input,output\} sets through it and adjusting the weights to minimize the sum of the squares of the differences between the desired result and the computed result. This quadratic function is the so-called energy, cost, or objective function. The weights are adjusted using what is known as the generalized delta rule [1]. The energy function, E, can be written

$$
\mathrm{E}=\underset{\mathrm{p}}{\sum} \sum_{\mathrm{i}}\left[\mathrm{t}_{\mathrm{i}}(\mathrm{p})-\mathrm{o}_{\mathrm{i}}(\mathrm{p})\right]^{2}
$$

where $t_{i}(p)$ is the value of the training output for output neuron $i$ and training data se' $p$; $\mathrm{o}_{\mathrm{i}}(\mathrm{p})$ is the corresponding network output. $\mathrm{E}$ is minimized using an iterative procedure whereby the weights $w_{i j}$ are adjusted according to:

$$
\Delta \mathrm{w}_{\mathrm{ij}}=\underset{\mathrm{p}}{\sum} \eta \delta_{\mathrm{i}}(\mathrm{p}) \mathrm{o}_{\mathrm{j}}(\mathrm{p})
$$

where $\quad \delta_{i}(p)=\left[t_{j}(p)-o_{i}(p)\right] o_{i}(p)\left[1-o_{i}(p)\right]$ for output layer neurons,

and $\quad \delta_{i}(p)=o_{i}(p)\left[1-o_{i}(p)\right] \underset{j}{\sum} w_{i j} \delta_{j}(p)$ for hidden layer neurons.

The parameter $\eta$ is called the training rate coefficient. We allow the user to use a different value of $\eta$ for the hidden and output layers. The weights are adjusted starting with the 
neurons in the output layer and moving back a layer at a time toward the input layer. Although the above is the standard delta rule algorithm we use the following, based on a suggestion by Chen and Mars [2], for the weight adjustment to the output layer:

$$
\delta_{i}(p)=t_{i}(p)-o_{i}(p)
$$

We also incorporate so-called "threshold neurons" which have values fixed at +1 and which are added to the input and hidden layers. In addition we use "momentum" in the training algorithm where a fraction $\alpha \delta_{j}$ of the previous weight adjustment is added to the new weight adjustment. The quantity $\alpha$ is a user provided input to the program.

The ANN will assimilate and correlate data given it in the process of "training" so that when given input vectors outside of the training set it will produce reasonable values for the corresponding output vectors. The matrix of weights, $w_{i j}$, clearly represents the mapping between the set of input vectors and the set of output vectors and contains all the information correlating the output to the input. 


\section{THE PSNN CODE}

\subsection{Program Summary}

The PSNN program is written in double precision in a version of contemporary Fortran that we have found to be available on most computers. The program is self-contained in two files and uses no external subroutine libraries such as IMSL or NAG. It has been tested on 80386 and 80486 personal computers using the Lahey F77L-EM/32 compiler and on HP, DEC, IBM, and Sun workstations.

The program uses arrays that are dimensioned using PARAMETER statements; these are easily changed using any good editor. Much of this could be simplified in the future as MAKE and INCLUDE utilities become more standardized and common to all computer systems. In this version of PSNN, some of the I/O are ASCII files; the file containing the information for restarting and for production runs is in a binary format.

The sections that follow contain information about the input and output units and files; the input data structures; the contents of the output files; and the variables and dimensions within the program. 


\subsubsection{I/O Units and Files}

The input and output units, filenames, and descriptions are listed below. A "problem" is defined uniquely by a name, and the various files are identified by extensions to that name:

\section{Input Units}

Unit \#

4

4

4

5

File Name (extension)

inp

.wts

dat

Output Units

\section{Unit \#}

4

6

7

10
File Name (extension)

.wts

. $\mathrm{nn} 7$

. n10

\section{Comments}

training facts

weights, etc. for trained

network and restarting

"unknown" input data

console input

\section{$\underline{\text { Comments }}$}

weights, etc. for trained network and restarting console output training details for each input pattern table of output neuron errors for all training patterns for use in doing statistics, histograms, graphics, etc. 


\subsection{Input to PSNN}

The user provided input to PSNN consists of one ASCII file (of training data or "unknown" data) and the user responding to a series of questions asked by the code at initiation. The format of the ASCII training data (input patterns) is outlined below. The architecture of the network and training parameters are described by the user's response. For a network that has already been trained, the ASCII file contains the input patterns for which the network is supposed to compute an output. The ASCII training pattern file is in free format so that for each pattern it consists of a list of values for the input neurons followed by the list of target output values. The name of the pattern file is provided by the user during the initial question/answer period.

The input patterns are line intensities. In developing the code we used the nine lines $\mathrm{H}(\alpha, \beta, \gamma, \delta)$ and $\mathrm{He}(\mathrm{IC}, \alpha, \beta, \gamma, \delta)$ but the ionization states and line identifications are not relevant to the operation of the code. The output neurons are:

(1) $\mathrm{T}_{\mathrm{e}}(\mathrm{eV})$

(2) $\quad \mathrm{N}_{\mathrm{e}}\left(\mathrm{cm}^{-3}\right)$

(3) $\mathrm{N}(\mathrm{He}) /[\mathrm{N}(\mathrm{H})+\mathrm{N}(\mathrm{He})]$; input is actually $\mathrm{N}(\mathrm{He}) / \mathrm{N}(\mathrm{H})$ but is

converted to this. The operation of the code does not depend on these identifications of the output neurons, but the printed output uses these labels. A sample of the pattern file that we used in developing the program is shown below:

$$
\begin{aligned}
& \begin{array}{lllll}
3.377 \mathrm{E}-19 & 6.187 \mathrm{E}-23 \quad 1.959 \mathrm{E}-24 & 3.107 \mathrm{E}-25 & 6.743 \mathrm{E}-01 \quad 1.000 \mathrm{E}+00 \quad 6.657 \mathrm{E}-03
\end{array} \\
& 2.463 \mathrm{E}-043.363 \mathrm{E}-05 / \mathrm{H}(\alpha, \beta, \gamma, \delta) \& \mathrm{He}(\mathrm{IC}, \alpha, \beta, \gamma, \delta) \text { in arbitrary units }
\end{aligned}
$$

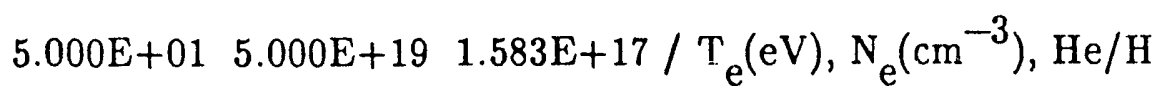


These line intensities were computed for an Al plasma by the code RATION [3] for the following ranges of the independent variables:

$$
\begin{aligned}
& 500 \leq \mathrm{T}_{\mathrm{e}} \leq 1200 \mathrm{eV} \\
& 5 \times 10^{19} \leq \mathrm{N}_{\mathrm{e}} \leq 6.4 \times 10^{21} \mathrm{~cm}^{-3} \\
& 1.6 \times 10^{17} \geq \mathrm{N}(\mathrm{He}) / \mathrm{N}(\mathrm{H}) \geq 6.5 \times 10^{-2}
\end{aligned}
$$

The network had difficulty in dealing with a 19 order of magnitude range in the ionization balance so within the program this is converted to the He fraction as has been noted above.

Upon executing the code, the user is asked if it is an initial trainiug run, a continuation training run, or a production run.

When the network is first trained for a new problem, the first user's response is a general purpose comment that identifies the problem. The the second response is the problem name whose ASCII training pattern file ends with the extension inp. The code then asks for a description of the network architecture. The default training parameters are then presented and the user may change them if he/she so desires. The key parameters and their default values are listed in section 2.4.2.

The input quantity "eps" has the following meaning. We renormalize the input and output values to the interval $(0,1)$ but do not want the minimum value to be exactly zero or the maximum value to be exactly one. So after finding the minimum and maximum 
values, say $x_{\min }$ and $x_{\max }$, the renormalization is performed using an $x_{\min }^{\prime}$ and $x_{\max }^{\prime}$ defined by:

$$
x_{\min }^{\prime}=(1-\epsilon) x_{\min } \text { and } x_{\max }^{\prime}=(1+\epsilon) x_{\max }
$$

This program is entirely self-contained and, hence, has its own random number generators. If the quantity "iseed" is equal to -1 , the random number generator always uses the same seed. If "iseed" is greater than zero the random number generator is called "iseed" times in a loop at the beginning of the program so that by changing "iseed" the user can change the random number sequence.

The user may change the mean, "gmu," and standard deviation, "gsigma," for the Gaussian distribution of the initial values of the weights. The training rate parameters are "etao" and "etah" for the output and hidden layers, and the momentum multiplier is "alpha." The global training criterion is "errcrit0;" the network is considered to be trained to that level when the absolute fractional difference between the target outputs and the network outputs is less than this criterion for all patterns.

The user has the option of training the network with noisy input data. If the input variable "noise" is not zero the input values are multiplied by

$$
1+\left(\mu_{\text {noise }}+\sigma_{\text {noise }} \xi\right)
$$

where $\xi$ is a standard Gaussian distributed random number.

Finally the user may specify the number of passes that the network is to make through the training pattern set, how often the (short) output is written to the console and 
how often the long output (to the file with extension .nn7) is to be written.

\subsection{Output From PSNN}

There are several output files generated by PSNN, one of which is used for future runs, i.e., restarts and operation as a trained network; this file has the extension .wts. An example of the short (console) ouiput, which is meant to be a summary of the training progress, is shown below. It displays the number of training patterns that the network successiully matched to within the error criterion, the number of incorrect outputs, the cycle on which the network got the most correct, the value of the energy function, and error statistics for each of the output neurons. These last consist of the best match of the network outp't to the target output, the average error over all patterns, the worst match, and the number of patterns that were successfully matched by that neuron.

\section{Console Output}

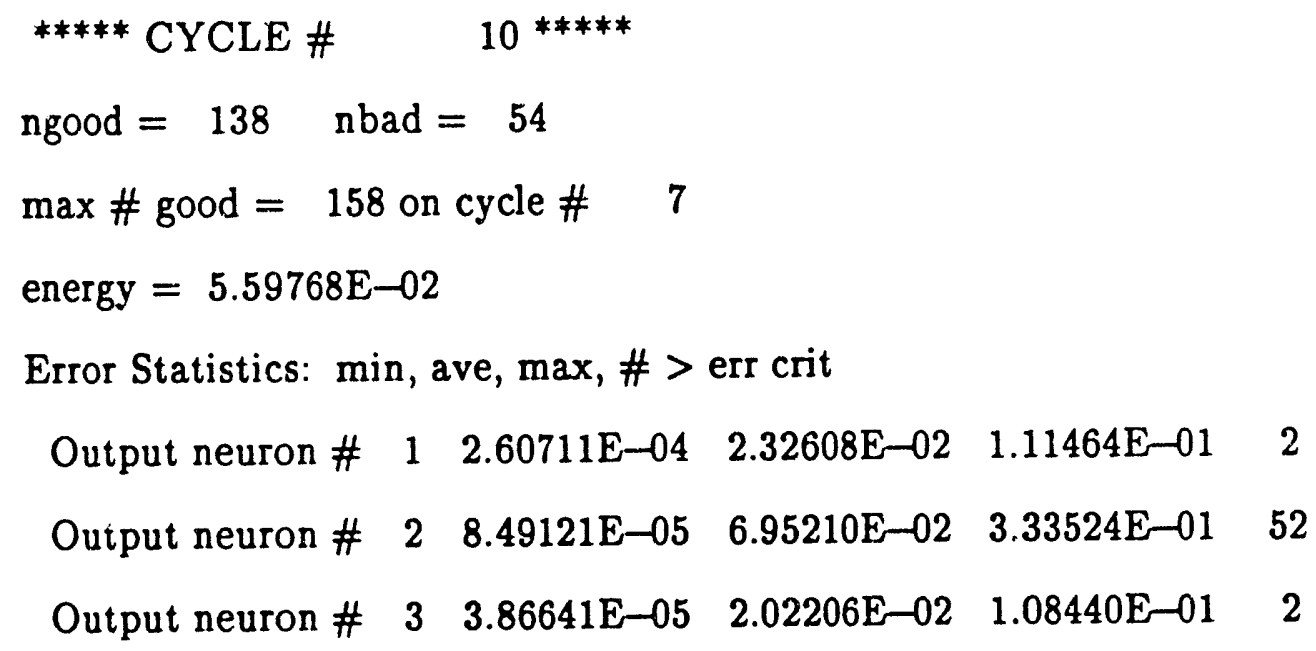

The ASCII output file .nn7 contains details of the training progress for each pattern. The training patterns are listed in the shuffled order that they were fed into the network but the correct sequence number for the training data input file is shown in parenthesis. 
The output is listed as "good" or "bad" depending on whether or not the network output for that pattern was within the error criterion. For each output neuron is shown the target value, the network output value, and the fractional error. A sample of part of this output file is shown below.

\section{Unit 7 Output}

ip $=47(156)$

good

Training pattern, network output, error:

Te: $\quad 1000.00000000000 \quad 1001.14260942602 \quad-0.114260942601563 \mathrm{D}-002$

Ne: $\quad 0.400000000000000 D+021 \quad 0.383936551534282 D+021 \quad 0.401586211642947 D-001$

$\mathrm{He} /(\mathrm{H}+\mathrm{He}): \quad 0.104022937012812 \quad 0.103896381860794 \quad 0.121660814097841 \mathrm{D}-002$

ip $=48(\quad 83)$

bad

Training pattern, network output, error:

Te: $\quad 550.000000000000 \quad 549.787876472408 \quad 0.385679141076173 \mathrm{D}-003$

$\mathrm{Ne}: \quad 0.200000000000000 \mathrm{D}+021 \quad 0.210496680778461 \mathrm{D}+021-0.524834038923069 \mathrm{D}-001$

$\mathrm{He} /(\mathrm{H}+\mathrm{He}): \quad 0.461235924788535 \quad 0.462462649067124 \quad-0.265964599169365 \mathrm{D}-002$

The other output file has extension .n10, which is a table of output neuron errors for all training patterns. This last has been useful in doing statistics on the errors and was used in making Figs. 2 and 3 in Section 3 below. 


\subsection{Programming Details of PSNN}

\subsubsection{Dimensions and Parameter Statements}

The array dimensions that appear in PARAMETER statements in the program are the following:

$$
\begin{aligned}
& \text { NLX }=\max \text { number of layers } \\
& \text { NHLX }=\max \text { number of hidden layers }=\text { NLX }-2 \\
& \text { NIN }=\text { max number of neurons in input layer } \\
& \text { NHN }=\text { max number of neuro. } 2 s \text { in hidden layer } \\
& \text { NON }=\text { max number of neurons in output layer } \\
& \text { NTP }=\max \text { number of training patterns }
\end{aligned}
$$

The layers of the neural network are numbered as follows:

$$
\begin{aligned}
& 1=\text { input layer of neurons } \\
& 2 \text { through (nlayers-1) }=\text { hidden layers } \\
& \text { nlayers = output layer }
\end{aligned}
$$

\subsubsection{Key Variables in the Program}

The key variables used in the program are defined as follows:

$$
\begin{aligned}
& \text { ni0, no0 = number of input \& output neurons } \\
& \text { nhlayers = number of hidden layers } \\
& \text { nhlo(i) = number of neurons in each hidden layer } \\
& \text { gmu, gsigma = parameters for initial Gaussian distribution of weights } \\
& \text { errcrit } 0=\text { training criterion (presently global for all output neurons) }
\end{aligned}
$$




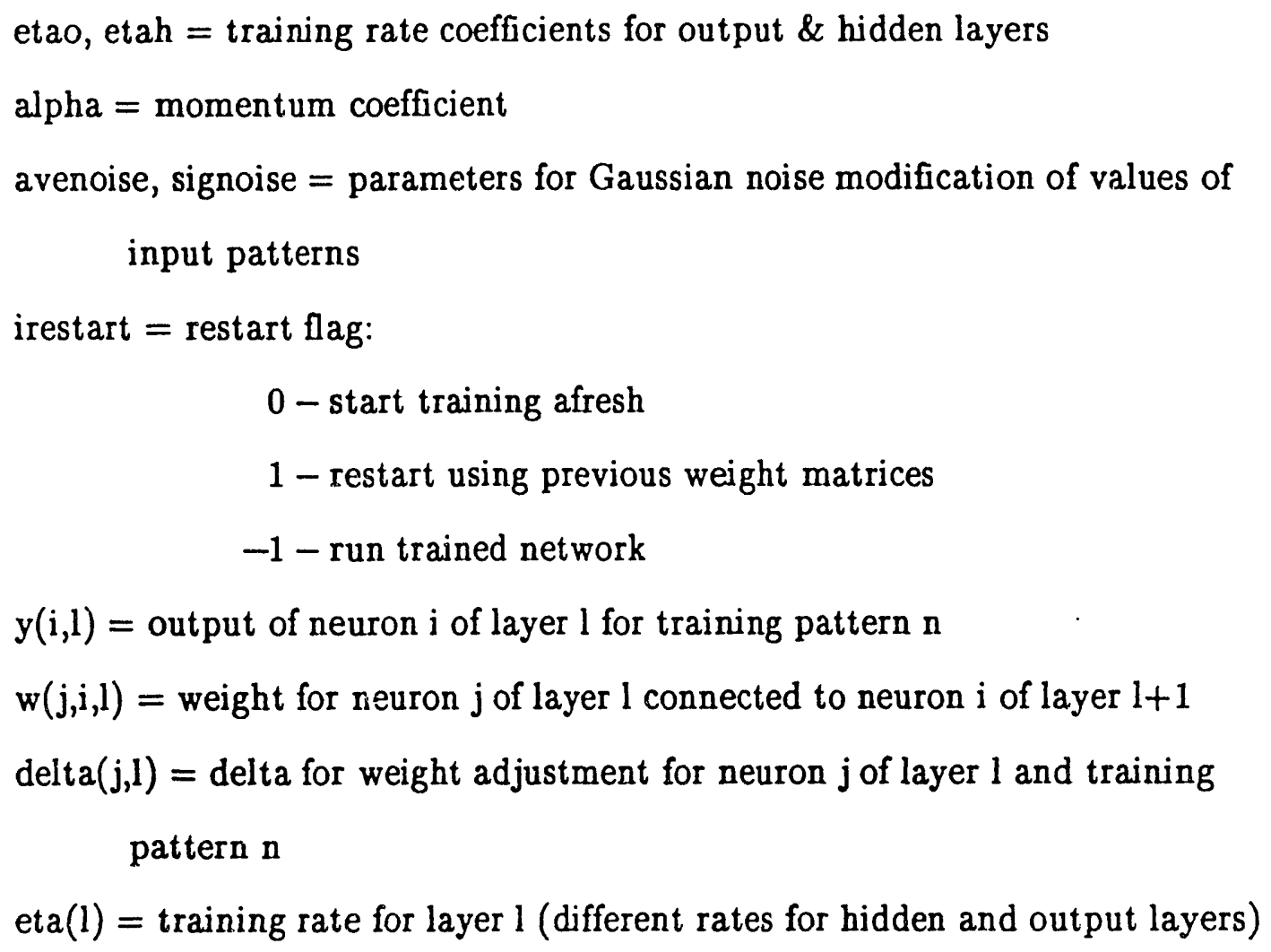

\subsubsection{List of Subroutines and Their Functions}

\section{PSNN Subprograms}

(in the order in which they appear in the program)

\section{INPUT:}

This routine reads the console (network definition) and unit 4 (training facts) input files.

FFBP:

This is the feed-forward, back-propagation training routine. 
NEURON:

This performs the operation:

$$
o_{i}=T\left(\sum_{j} w_{i j} o_{j}\right)=1 /\left(1+e^{-\sum w_{i j} j_{j}}\right)
$$

for the output from neuron $i$ based on the weighted outputs from neurons $j$ in the previous layer.

FEEDFOR:

This is the feed-forward loop for a trained network.

PREPARE:

This routine performs the normalizations of the input \& output patterns.

SIGNUM:

This provides the sign of the argument $x$. It is -1 if $x<0$ and +1 if $x \geq 0$.

INITWTS:

This routine generates the random initial weight matrices from a Gaussian distribution with mean $\mu$ and standard deviation $\sigma$, which are inputs to the program.

\section{TABLE3D:}

This propagates a constant into all elements of a $3-D$ array.

VMAXMIN:

This routine finds the maximum and minimum of a vector. 
NSHUFFLE:

This routine shuffles an vector of integers $1 \leq \mathrm{v} \leq \mathrm{n}$ and is used for shuffling the training patterns on every pass through the back-propagation process.

RAN1:

Uniform random number generator taken from Press, et al., Numerical Recipes [4].

\section{GASDEV:}

Gaussian random number generator taken from Press, et al., Numerical Recipes [4].

\section{SETFT:}

Sets up the various filename extensions. 


\section{USERS GUIDE AND EXAMPLES}

In order to use the code the dimensions should be made appropriate to the problem, although a diagnostic will be issued if they are not, and then it should be compiled. The Fortran compile line on the HP workstation, for example, is:

$$
\mathrm{f} 77 \text { - } \text { net.x }-\mathrm{K}-\mathrm{R} 8+\mathrm{O} 3+\mathrm{OS} \text { psnn.f }
$$

In doing timings on various computers we found that the network with 9 input neurons, 3 output neurons, and 2 hidden layers of 15 neurons each training on 192 patterns took 112 seconds for 1000 passes through the training data on an HP-730 and 255 seconds on a DEC-5000. To help assess the relative speeds of various machines Table 1 shows timings for a generic Molecular Dynamics code that has been run on several computers.

Fig. 2 shows a summary of the training of the network to the $10 \%$ level. First the training criterion was set at $30 \%$ with $\eta_{0}=0.9, \eta_{\mathrm{h}}=0.5$, and $\alpha=0.9$. It took 800 cycles, i.e. passes of the full set of training patterns through the network, to converge to the $30 \%$ level. The criterion was then set to $20 \%$ with the training parameters the same and the network then converged to this error value in 1694 cycles after the restart. The error criterion was then set to $10 \%$ and the network was restarted with the same training parameters for another $10^{4}$ cycles. It was then run for another $10^{4}$ cycles with $\eta_{0}=0.5, \eta_{\mathrm{h}}=0.3$, and $\alpha=0.5$, and then for another 4309 cycles with the training parameters being $0.2,0.1$, and 0.2 respectively until it converged. The progress of training can be seen in Fig. 2. Most of the difficulty in training had to do with the second neuron, i.e. $\mathrm{N}_{\mathrm{e}}$. The training parameters were reduced in order to avoid the possibility of oscillating about the minimum in the functional hypersurface rather than dropping into it. 
This network was eventualiy trained to the $5 \%$ level. The average error in $\mathrm{T}_{\mathrm{e}}$ and ionization balance was about $1 \%$ with maximum errors of about $4 \%$. The histogram in Fig. 3 shows the distribution of errors for the 192 training patterns for the second output neuron, i.e. $\mathrm{N}_{\mathrm{e}}$. The average is $1.8 \%$ and the maximum is just about $5 \%$. 


\section{REFERENCES}

\subsection{References Cited Above}

1. J. Hertz, A. Krogh, and R.G. Palmer, Introduction to the Theory of Neural Computation, (Addison-Wesley, Redwood City, CA, 1991).

2. D. Tveter, AI Expert, July 1991, p. 36.

3. R.W. Lee, B.L. Whitten, and R.E. Strout II, JQSRT 32, 91 (1984).

4. W.H. Press, B.P. Flannery, S.A. Teukolsky, and W.T. Vetterling, Numerical Recipes, (Cambridge Univ. Press, Cambridge, 1986).

\subsection{Bibliography}

R. Hecht-Nielsen, Neurocomputing, (Addison-Wesley, Reading, MA, 1990).

G. E. Hinton and T. J. Sejnowski, "Learning and relearning in Boltzmann machines," in Parallel Distributed Processing, vol. 1, ed. D.E. Rumelhart and J. L. McClelland, (MIT Press, Cambridge, MA, 1986), pp. 282-317.

J.J. Hopfield, Proc. Nat. Acad. Sci. 79, 2254 (1982); 81, 3088 (1984).

J.J. Hopfield and D.W. Tank, Biological Cybernetics 52, 141 (1985).

B. Kosko, Neural Networks and Fuzzy Systems, (Prentice Hall, Englewood Cliffs, NJ, 1992).

A. Lapedes and R. Farber, "How neural nets work," in Neural Information Processing Systems, ed. D.Z. Anderson, (Amer. Inst. of Physics, New York, 1988), pp. 442-456. 
W.L. Morgan, IEEE Trans. on Plas. Sci. 19, 250 (1991).

P. D. Wasserman, Neural Computing, (Von Nostrand Reinhold, New York, 1989). 


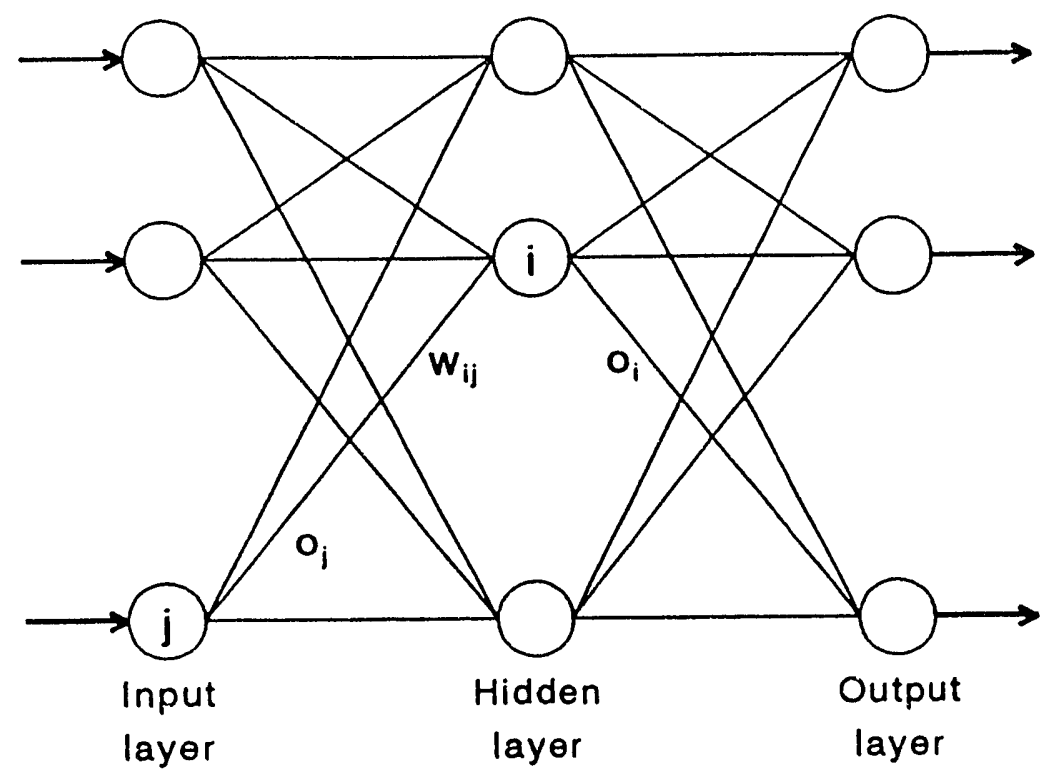

Figure 1: Three-layer feedforward, backpropagation neural network. The neurons, denoted by circles, in a given layer operate via a transfer function on the weighted output from the neurons in the previous layer to produce an output signal that is passed on to the next layer.

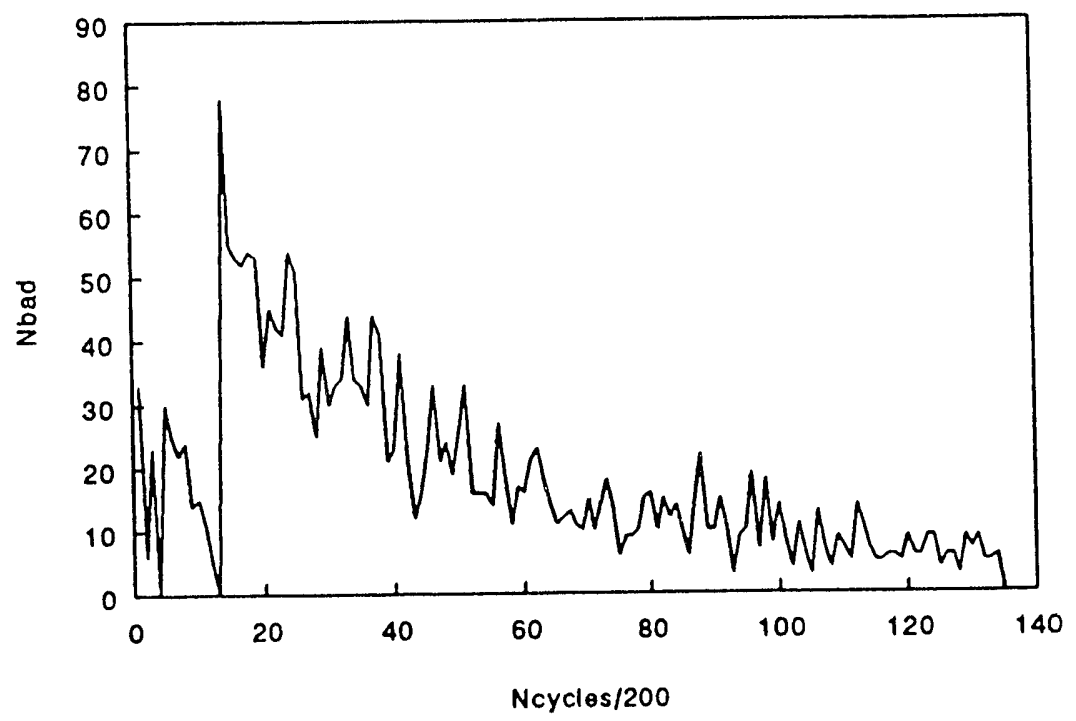

Figure 2: The number of patterns for which the neural network failed to match the target output as a function of the number of passes (Ncycles). 


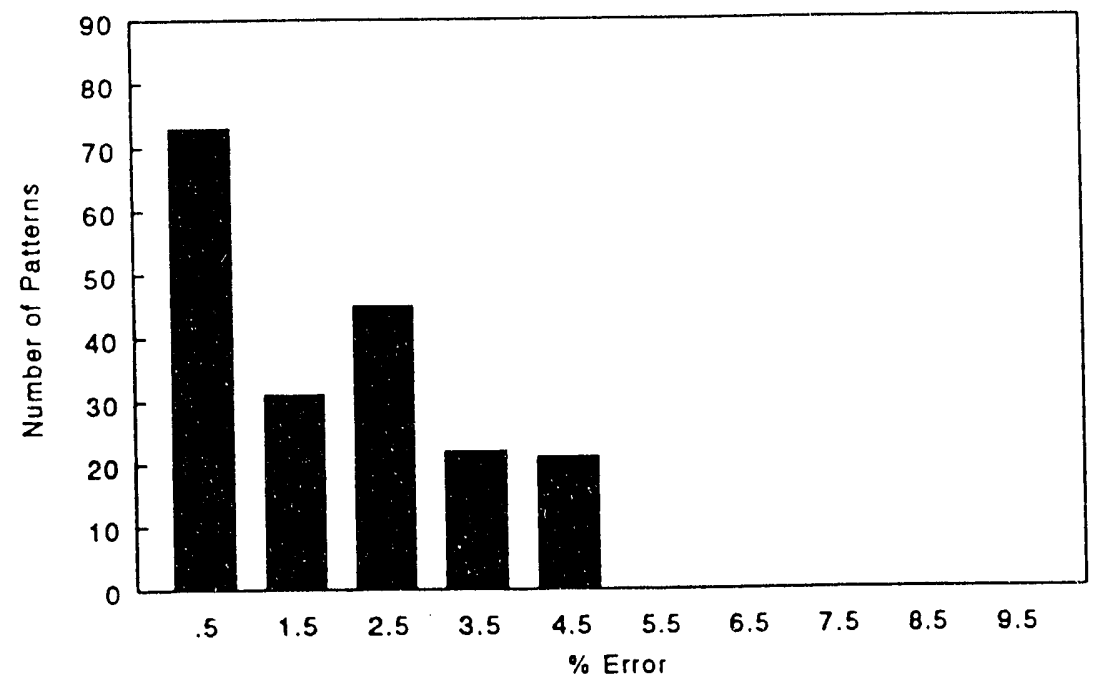

Figure 3: Histogram of fractional difference between network output and training target output for $N_{e}$.

Computer

$80386 / 8725 \mathrm{MHz} \mathrm{PC}$ $80486 \quad 33 \mathrm{MHz}$ PC

VAX 3900

VAX 6400

Sun 4 workstation

DEC $/ 5000$ workstation

IBM RISC/6000 workstation

HP 730 workstation (full optimization)

Cray X-MP

Cray $\mathrm{Y}-\mathrm{MP}$ unvectorized vectorized unvectorized vectorized
Time (sec)

180

105

78

37

42

24

16

7.4

18

3.5

10

1.6

Table 1: Relative speeds of various computers. 

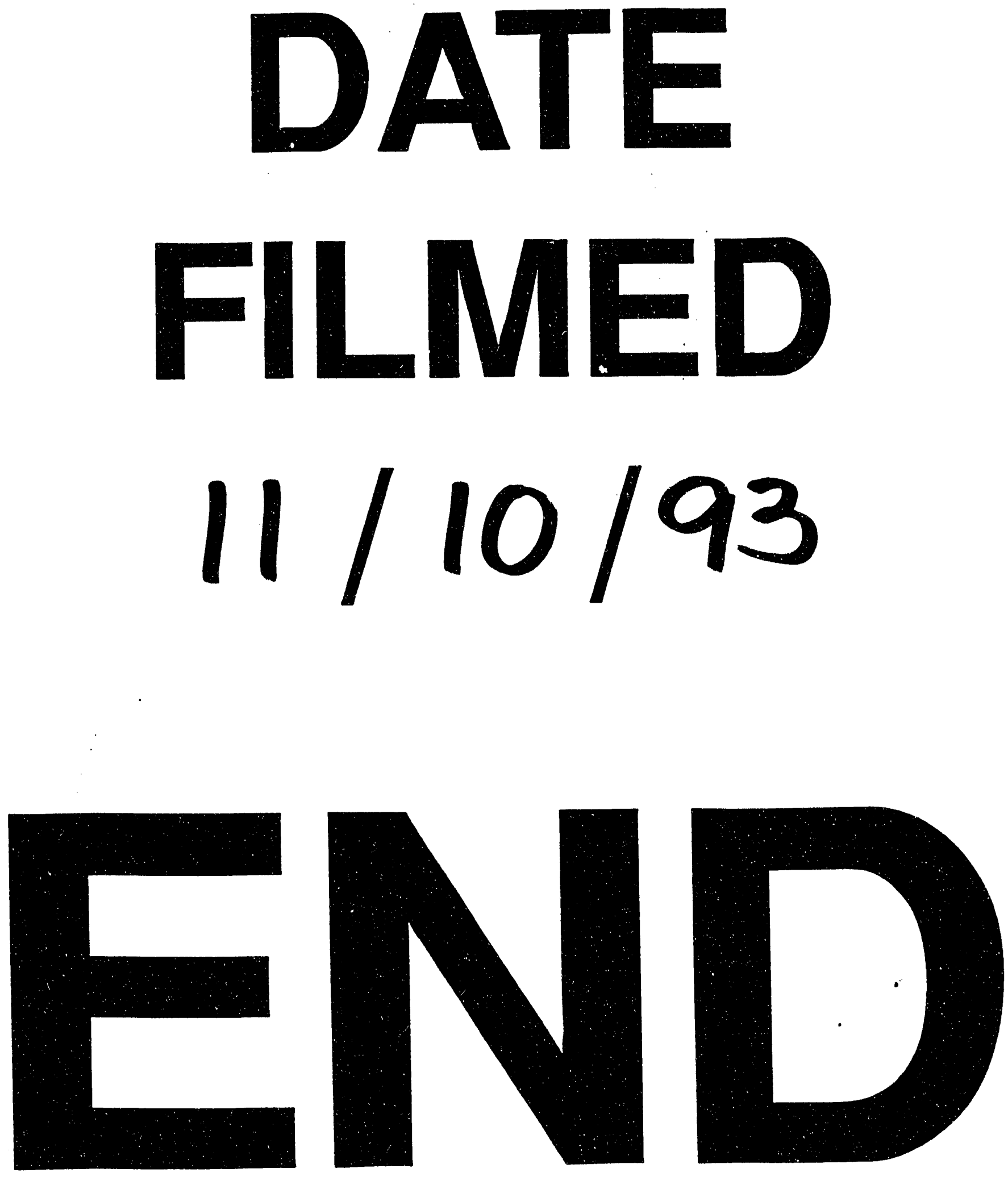
